Bundesgesundheitsbl - Gesundheitsforsch Gesundheitsschutz 2003 • 46:694-699 DOI 10.1007/s00103-003-0652-z

Forschung aktuell

A. Flieger · S. Banerji · M. Broich · K. Rydzewski · W. S. Shadrach

Robert Koch-Institut, Berlin

\title{
Pathogenese der Legionelleninfektion
}

\section{Arbeiten der Nachwuchsgruppe „Pathogenese der Legionelleninfektion" des Robert Koch-Instituts}

\section{Geschichtliches}

Legionellen sind Gram-negative Bakterien, die im Süßwasser als intrazelluläre Parasiten von Protozoen vorkommen. Nach Inhalation besiedelt das Bakterium Alveolarmakrophagen und alveoläre Epithelzellen der Lunge. Beim Menschen kann insbesondere die Spezies Legionella pneumophila die Legionärskrankheit, eine schwere Pneumonie, auslösen, die oft einen Funktionsverlust der Lunge zur Folge hat. In den letzten Jahren wurde eine Reihe von bakteriellen Faktoren beschrieben, die die intrazelluläre Vermehrung und Virulenz fördern. Unter diesen befindet sich das Typ-II-Protein-Sekretionssystem Lsp von L. pneumophila, das hydrolytische Enzyme, wie z. B. die Zink-Metalloprotease, saure Phosphatasen, Phospholipasen A und Lysophospholipasen A transportiert. Im Moment ist noch nicht vollständig geklärt, welche dieser exportierten Faktoren die eigentlichen Effektoren von intrazellulärer Vermehrung und Virulenz sind. Phospholipase-Aktivität wurde bei Bakterien (z.B. Pseudomonas aeruginosa, Listeria monocytogenes, Yersinia enterocolitica) als einer der maßgeblichen Pathogenitätsfaktoren charakterisiert. Somit könnte die Phospholipase-A-Aktivität von L. pneumophila z.B. über die Zerstörung von alveolärem Lungenepithel, Alveolarmakrophagen und Lungensurfactant zur Pathogenese einer Legionelleninfektion beitragen. Die Forschungsvorhaben der Nachwuchsgruppe "Pathogenese der Legionelleninfektion" haben zum Ziel, neue Pathogenesemechanismen von Legionellen zu charakterisieren und sollen gleichzeitig versuchen, neue Therapiemethoden über die Inhibition von hydrolytischen Enzymen zu etablieren.
1976 erkrankten bei einem Treffen von ca. 4.400 Veteranen der American Legion in einem Hotel in Philadelphia (USA) 182 Teilnehmer an einer bisher unbekannten Form der Lungenentzündung, und 34 der Erkrankten verstarben. Innerhalb von 6 Monaten nach dem Ausbruch der Krankheit wurde ein Gram-negatives Stäbchenbakterium, das keiner der bekannten Bakterienspezies zugeordnet werden konnte, als Erreger der Erkrankung isoliert. Das Bakterium benannte man nach der hier betroffenen Gruppe der Erkrankten und der Lokalisation des Erregers in der Lunge Legionella pneumophila $[1,2]$. Anhand eingefrorener Patientenseren konnten auch frühere Ausbrüche (der Älteste geht auf 1947 zurück) mit ähnlicher Symptomatik auf Legionellen zurückgeführt werden [3]. In den folgenden Jahren wurden weitere Legionellenspezies isoliert. Heute besteht der Genus Legionella aus mehr als 46 verschiedenen Spezies. Von L. pneumophila, der am häufigsten mit einer Legionellose assoziierten Spezies, sind 14 Serogruppen bekannt [4].

\section{Reservoire für Legionellen}

Legionellen sind Keime, die in natürlichen und künstlichen feucht-wässrigen Umgebungen, wie z. B. Duschköpfen, Klimaanlagen (dies war auch das Reservoir für die Infektionen in Philadelphia 1976), Kühltürmen, Flüssen, Seen und sporadisch auch in feuchtem Boden vorkommen. Sie können sowohl als intrazellulärer Parasit von Protozoen (z. B. Amöben der Gattungen Hartmannella oder Acanthamoeba,
Abb. 1), in Assoziation mit Biofilmen oder auch freilebend auftreten $[4,5,6]$.

\section{Klinische Aspekte der Legionärserkrankung}

Legionellen werden über keimhaltige Aerosole (Vernebelung von Wasser z. B. durch Duschköpfe) vom Menschen eingeatmet und gelangen so in die Lunge. Ob es zu einer Erkrankung nach Legionellenkontakt kommt und wie schwer diese verläuft, hängt von der Quantität der eingeatmeten Bakterien, ihrer Assoziation mit Amöben und auch vom Immunstatus des möglichen Wirtes ab $[7,8]$. Es sind 2 verschiedene Formen des Krankheitsverlaufes einer Legionelleninfektion bekannt: 1) leicht und grippeähnlich, Pontiac-Fieber genannt, und 2) schwer und oft in eine lebensbedrohliche Pneumonie mündend, die Legionärskrankheit. Die Legionärskrankheit hat eine Inkubationszeit von 2-10 Tagen. Erste Anzeichen dieser Erkrankung sind u. a. Müdigkeit, Benommenheit, Fieber (oft über $40^{\circ} \mathrm{C}$ ), Husten, Kopf- und Gliederschmerzen. Radiologisch beobachtet man alveoläre, fleckenförmige Verschattungen der Lunge [9]. Die Lungenbläschen sind mit neutrophilen Granulozyten, Makrophagen, Fibrin und Erythrozyten angefüllt [10]. Aufgrund des Funktionsverlustes der Lungenbläschen (z. B. durch Verstopfung) kann die Legionellenpneumonie in ein ARDS (,acute respiratory distress syndrome“) münden [11].

(c) Springer-Verlag 2003

Dr. A. Flieger

Robert Koch-Institut, Nordufer 20,13353 Berlin E-Mail:fliegera@rki.de 

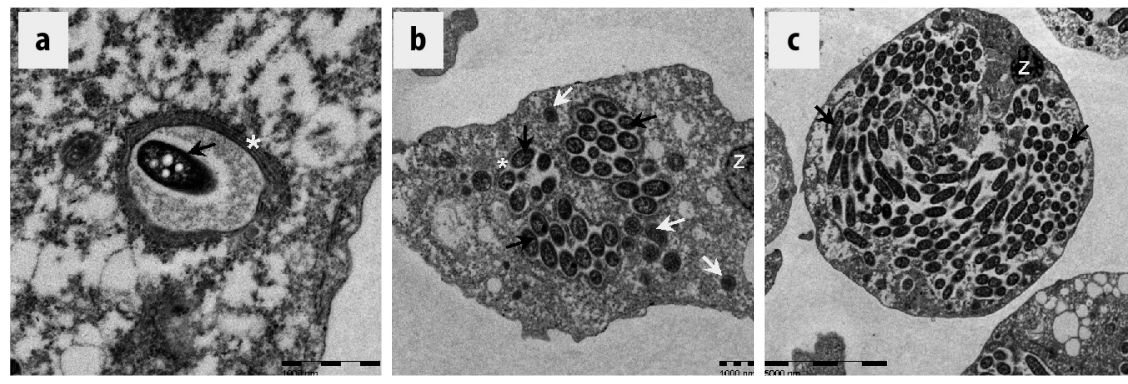

Abb. 1a-c $\Delta$ Elektronenmikroskopische Aufnahmen von Hartmannella-vermiformis Amöben, die mit L. pneumophila infiziert wurden. a Charakteristisches Legionellen-Phagosom mit einer Bakterienzelle (schwarzer Pfeil), das von endoplasmatischem Retikulum umgeben ist (weißer Stern). b Mehrere Legionellen-enthaltende Phagosomen in Hartmannella vermiformis. Die intrazelluläre Vermehrung des Bakteriums (schwarze Pfeile) hat begonnen. Mitochondrien (weiße Pfeile) befinden sich in der Nähe der Legionellen-Phagosomen. Die Phagosomen sind von endoplasmatischem Retikulum umgeben (weißer Stern). c Fortgeschrittenes Stadium der Infektion von Hartmannella vermiformis mit Legionellen. Der zytoplasmatische Raum einer Amöbe ist fast vollständig von einem vor Legionellen (schwarze Pfeile) berstenden Phagosom ausgefüllt. (Z Zellkern)

Durch Legionellen werden bis $\mathrm{zu}$ 22,5\% aller ambulant erworbenen und bis zu $40 \%$ aller nosokomialen Pneumonien verursacht [12]. Für das Jahr 2002 wurden in Deutschland 413 Fälle an Legionellose gemeldet [13]. Gemessen an der Zahl der Legionellosen in anderen Ländern (USA) wird jedoch angenommen, dass die eigentliche Zahl an Legionellen-assoziierten Erkrankungen ein Vielfaches der gemeldeten Fälle beträgt.

\section{Etablierung einer Legionellen- infektion - zelluläre und molekulare Aspekte}

Wenn bakterienhaltige Aerosole eingeatmet werden und somit in die unteren Atemwege der Lunge gelangen, werden die Bakterien anschließend von Alveolarmakrophagen phagozytiert (Abb. 2). Im Makrophagen liegt L. pneumophila in einem besonderem Phagosom vor, das von Wirtszellorganellen (Mitochondrien, endoplasmatisches Retikulum) umgeben ist und in dem sich die Bakterien vermehren können, bis das Phagosom fast die gesamte Wirtszelle ausfüllt (s. Abb. 1c, Abb. 3) [14]. In der anfänglichen Wachstumsphase verhindern die Bakterien eine Ansäuerung und Reifung des Phagosoms [15]. In Mausmakrophagen wurde gezeigt, dass zu späteren Zeitpunkten die Mehrheit der Legionella-Phagosomen lysosomale Marker, und der $\mathrm{pH}$-Wert im Phagosom auf 5,5 absinkt [16]. L. pneumophila vermehrt sich auch in diesem sauren, nährstoffreichen Phagolysosom [16]. Nach dem Verbrauch der Nährstoffreserven wandeln sich die Bakterien in ihre virulente Form (gekennzeichnet durch z.B. Motilität durch Flagellenexpression, Zytotoxizität) um, lysieren die Wirtszelle und beginnen einen neuen Infektionszyklus bei dem auch alveoläre Epithelzellen betroffen sein können (s.Abb.2) [17]. So kann es zur Zerstörung von Alveolarepithel kommen, das dann den Eintritt von Substanzen oder zellulärem Material aus dem angrenzenden Kapillarraum zulässt und somit zu einer Verstopfung der Lungenbläschen führt. Zusätzlich können durch die Lyse von Wirtszellen freigesetzte eukaryontische oder bakterielle hydrolytische Enzyme einen Abbau des Lungensurfactantfilms verursachen (s. Abb. 2). Intakter Lungensurfactant, der zu 80\% aus Phospholipiden besteht, ist vor allem für die Stabilität der Lunge (Verhinderung von Atelektasen) und für die Erniedrigung der bei der Atmung aufzuwendenden Arbeit wichtig [18]. Bei Patienten mit ARDS wurden typische Veränderungen im Phospholipidmuster und Störungen in der Funktion des Lungensurfactants gefunden $[19,20]$. Bisher wurde jedoch noch nicht gezeigt, ob dies auch für eine Legionellose zutrifft.

\section{Virulenzfaktoren von Legionella pneumophila}

Die Fähigkeit zur intrazellulären Vermehrung in Makrophagen wird als eine wichtige Virulenzdeterminante von Legionellen angesehen [4]. Im Genom von L. pneumophila sind bereits mehrere Genbereiche identifiziert worden, die im Zusammenhang mit der Fähigkeit zur intrazellulären Vermehrung stehen.
Dazu gehören z. B. der dot/icm-Genlocus, ein Typ-IV-Proteinsekretionssystem, und der $l s p$-Genlocus, ein Typ-IIProteinsekretionssystem [21, 22, 23, 24, 26]. Das Typ-II-Sekretionssystem von L. pneumophila exportiert hydrolytische Enzyme [23, 24, 25]. Der eigentliche TypII-sekretierte Faktor, der das intrazelluläre Überleben der Legionellen fördert, wurde bisher jedoch noch nicht identifiziert. Es ist daher von Interesse, die Beteiligung weiterer Typ-II-sekretierter Legionella-Proteine an der intrazellulären Replikationsfähigkeit von Legionellen näher zu untersuchen sowie neue sekretierte Effektoren aufzufinden.

\section{Phospholipasen \\ sind mikrobielle Virulenzfaktoren}

Phospholipase-Aktivität wurde z. B. bei pathogenen Bakterien (z. B. Pseudomonas aeruginosa, Listeria monocytogenes, Yersinia enterocolitica), als auch pathogenen Pilzen (z. B. Cryptococcus neoformans) oder Parasiten (z. B. Toxoplasma gondii) nachgewiesen und als einer der hauptsächlichen Pathogenitätsfaktoren dieser Mikroorganismen charakterisiert. Verschiedene Wirkungsweisen von Phospholipasen tragen zur Krankheitsentstehung bei:

D Mikrobielle Phospholipasen und ihre Reaktionsprodukte (z. B. Lysophosphatidylcholin) sind stark zytotoxisch, da sie die Integrität von zellabgrenzenden Membranen durch die Hydrolyse von Membranbestandteilen (Phospholipide) oder Porenbildung stören $[26,27]$.

D Phospholipasen verschiedener bakterieller Erreger sind in der Lage, die Lyse von Phagosomenmembranen zu unterstützen und damit zum intrazellulären Überleben der Bakterien oder nach Abschluss der intrazellulären Vermehrung durch die Lyse der Wirtszelle zur weiteren Verbreitung des Erregers beizutragen [28, 29].

D Die Reaktionsprodukte von Phospholipasen (z. B. 1,2-Diazylglyzerol, Arachidonsäure, Lysophosphatidylcholin) stellen wichtige Botenstoffe für eukaryonte Zellen dar [30,31].

D Das Phospholipase-A-Spaltprodukt Lysophosphatidylcholin ist ein multifunktionelles Lipid. Zusätzlich zu der früh beschriebenen zytotoxischen Eigenschaft des Lysophospholipids sind 


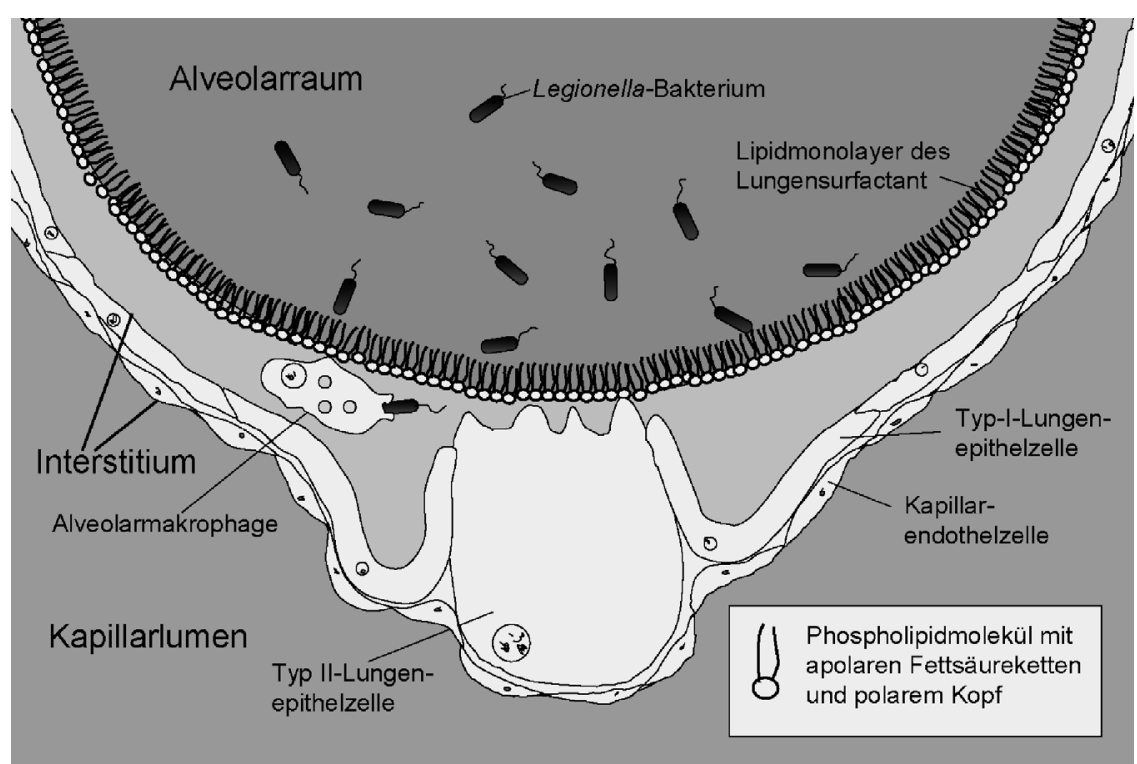

Abb. $2 \Delta$ Welche Umgebung finden Legionellen im Lungenbläschen vor? Schematische Darstellung eines Schnitts durch ein Lungenbläschen. Die Bakterien gelangen über die Aspiration von legionellenhaltigen Aerosolen in die Lungenbläschen und werden dort von Alveolarmakrophagen phagozytiert. Nach abgeschlossener intrazellulärer Vermehrung der Bakterien im Makrophagen, wird dieser lysiert (zum intrazellulären Zyklus von Legionellen s. Abb. 3). Ein Befall von weiteren Wirtszellen, wie z. B. auch Typ-Iund Typ-II-Lungenepithelzellen, ist möglich. Die Zerstörung von Lungenepithel, direkt durch die Infektion mit Legionellen und indirekt durch die Freisetzung von hydrolytischen Enzymen bakteriellen und eukaryonten Ursprungs nach Wirtszelllyse, kann die Dichtheit des alveolären Epithels gefährden, und dadurch kann eine Verstopfung der Lungenbläschen eintreten. Zusätzlich wird der Lungensurfactantfilm beeinträchtigt, der für die Funktion der Lunge notwendig ist

nun weitere Charakteristika entdeckt worden [26]. Lysophosphatidylcholin unterstützt die Entstehung von Entzündungen und besitzt eine Rolle in der Apoptoseinduktion [32,33].

- Außerdem sind die Phospholipide des Lungensurfactant, wie für Phospholipase-Aktivität von Pseudomonas aeruginosa gezeigt, ein gutes Substrat für diese Hydrolasen [34]. Die Zerstörung oder der Umbau der oberflächenaktiven Substanzen im Surfactantmaterial kann schwerwiegende funktionelle Störungen (Kollaps der Lungenbläschen und Erhöhung der Atmungsarbeit) in der Lunge hervorrufen [35].

Da viele Charakteristika einer Legionelleninfektion (Umprogrammierung von Wirtszellen, Apoptoseinduktion, Lyse aus der Wirtszelle, Zerstörung von Lungenzellen, Entzündungsentstehung, Verlust der Lungenfunktion) von einer Phospholipase-Wirkung herrühren könnten, jedoch der Zusammenhang zwischen Phospholipase-Aktivität und Pathogenität von L. pneumophila noch nicht untersucht wurde, begannen wir, Phospholi-
pase-Aktivität beim Lungenpathogen L. pneumophila zu erforschen.

\section{Analyse der Phospholipase- Aktivität von L. pneumophila}

\section{L. pneumophila sezerniert Phospholipase-A-Aktivität}

Als Erstes interessierte uns, ob L. pneumophila Phospholipase-Aktivität in das Wachstumsmedium sezerniert. Tatsächlich konnten wir nach Inkubation von L.-pneumophila-Kulturüberständen mit Phosphatidylcholin sowohlden Abbau diesesPhospholipidsalsauch die Bildung charakteristischer Phospholipase-A-Spaltprodukte wie Fettsäuren, Lysophosphatidylcholin und Glyzerophosphorylcholin nachweisen. Lysophosphatidylcholin wird dabei durch die Hydrolyse einer Fettsäure aus dem Phospholipidmolekül erzeugt, während Glyzerophosphorylcholin bei Abspaltung des 2. Fettsäurerrests entsteht (Abb.4) [36,37]. Da sowohl Lysophosphatidylcholin als auch Glyzerophosphorylcholin nachgewiesen wurden, vermuteten wir, dass die Phospholipidhydrolyse durch Kulturüberstände von L. pneumophila in
2 zeitlich aufeinander folgenden Teilschritten stattfinden muss (Abb. 4). In einem ersten Schritt katalysiert eine Phospholipase A (PLA) die Abspaltung einer Fettsäure aus einem Diazylphospholipid [enthält 2 (di) Fettsäureketten (azyl)], wobei ein Lysophospholipid gebildet wird. Im 2. Schritt wird die noch am Lysophospholipid befindliche Fettsäure durch eine Lysophospholipase A (LPLA) abgetrennt, und es entsteht Glyzerophosphorylcholin. Lysophospholipasen A sind Phospholipasen A, die präferenziell Fettsäureester von Lysophospholipiden hydrolysieren. Da wir unter unseren experimentellen Bedingungen bisher keine charakteristischen Spaltprodukte anderer Phospholipasen (wie Phospholipase C und D) finden konnten, stellt die neu beschriebene Phospholipase-A-Aktivität die hauptsächliche sekretierte Phospholipase-Aktivität von L. pneumophila dar $[36,37]$. Zelllysate des Bakteriums setzten ebenfalls Fettsäuren aus Phospholipiden und Lysophospholipiden frei, was auf das zusätzliche Vorhandensein von einer oder mehreren zellassoziierten Phospholipasen A bei L. pneumophila hindeutet [38].

\section{L.pneumophila sezerniert verschiedene Phospholipase-A-Aktivitäten}

Als Nächstes wollten wir prüfen, ob $L e$ gionella pneumophila verschiedene Phospholipasen A sezerniert, die jeweils einen Teilschritt (s. Abb. 4) der Hydrolyse von Phospholipiden katalysieren. Dazu führten wir eine Fraktionierung der im Kulturüberstand von L. pneumophila vorhandenen Proteine mittels Ultrafiltration (grobe Trennung nach Größe) und anschließenden chromatographischen Methoden (feine Trennung nach Eigenschaften, z. B. Ladung oder Größe) durch. In unterschiedlichen Fraktionen wurden dabei 5 verschiedenartige Phospholipase-A-Aktivitäten detektiert [39]. Während 3 dieser Aktivitäten nur Phospholipide mit zwei veresterten Fettsäuren (z. B. Phosphatidylcholin) hydrolysierten, war eine andere in der Lage, Fettsäuren nur aus Lysophospholipiden (z. B. Lysophosphatidylcholin) abzutrennen. Eine weitere Aktivität hydrolysierte sowohl Lysophospholipide als auch Diazylphospholipide [39]. Es konnten somit tatsächlich unterschiedliche Phospholipase-A-Aktivitäten nachgewiesen werden, die für eine Hydrolyse von Phospholipiden in 2 Teilschritten infrage kamen. 


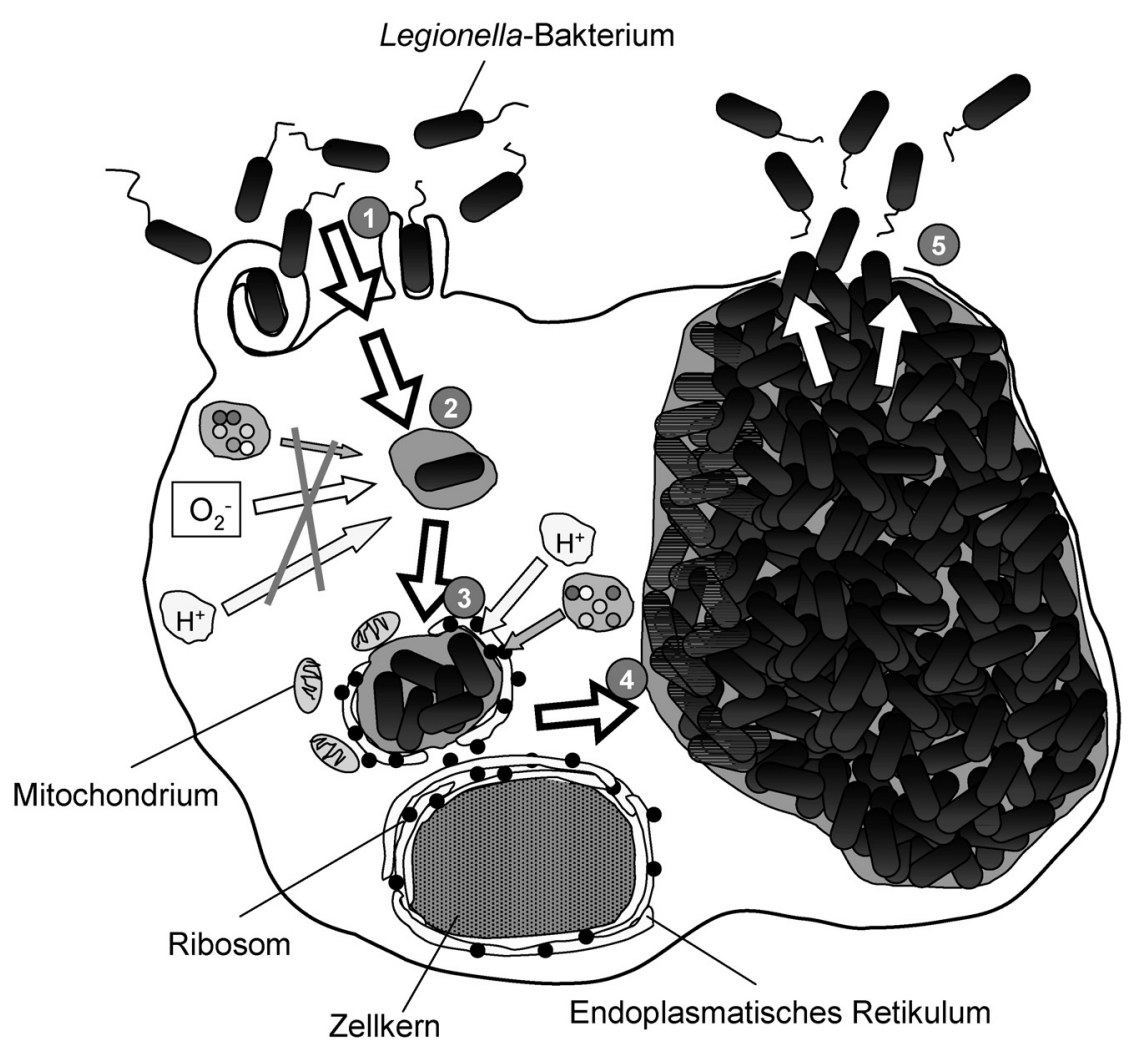

Abb. $3 \Delta$ Infektionszyklus von L. pneumophila im Makrophagen. 1) Entritt von Legionella-Bakterien in die Wirtszelle durch coiling- oder konventionelle Phagozytose.2) Blockierung des oxidative burst, der Ansäuerung des Legionella-Phagosoms und der Verschmelzung desLegionella-Phagosoms mit Lysosomen.3) Anlagerung von Wirtszellorganellen, wie Mitochondrien und endoplasmatischem Retikulum. Beginn der intrazellulären Vermehrung von Legionella im Phagosom. Im Laufe der Vermehrung wird das Phagosom angesäuert, und eine Verschmelzung mit Lysosomen findet statt. 4) Die Bakterien-Phagosomen wachsen auf eine Größe heran, sodass sie fast die gesamte Wirtszelle ausfüllen. 5) Nach Verbrauch der lokalen Nährstoffreserven findet eine Umwandlung der Bakterien in die virulente Form (flagelliert, zytotoxisch) statt. Durch Lyse der Phagosomen und Wirtszellmembran gelangen die Bakterien wieder in den extrazellulären Raum und können einen neuen Infektionszyklus initiieren

\section{Die Phospholipase-A- und die Lysophospholipase-A-Sekretion sind zeitlich verschoben}

Ein weiteres Indiz für die Sekretion von unterschiedlichen PLA- und LPLA-Aktivitäten wurde durch die Untersuchung von Sekretionskinetiken dieser Enzymaktivitäten gefunden. L. pneumophila sezerniert PLA- und LPLA-Aktivität von der mittleren logarithmischen Wachstumsphase an, wobei das Maximum an PLA-Aktivität vor dem Maximum der LPLA-Aktivität erreicht wird [40]. Bei Kontakt mit Phosphatidylcholin (in vivo z. B. in Form von Lungensurfactant) könnte L. pneumophila durch die zeitlich verschobenen Maxima von PLA und LPLA so das zytotoxische, multifunktionelle Lipid Lysophosphatidylcholin erzeugen und akkumulieren [40]. Andere Legionellen-Spezies, wie z. B. L. gormanii und L. steigerwaltii, erreichten das Sekretionsmaximum der LPLA-Aktivität vor der PLA-Aktivität, was bedeutet, dass Lysophosphatidylcholin-abbauendes (also entgiftendes) Enzym (LPLA) sofort bereitsteht, wenn PLA das zytotoxische Agens aus Phosphatidylcholin generiert [40]. Das Phänomen der zeitlichen Nachlagerung der Maxima von PLA vor LPLA könnte bei L. pneumophila ein entscheidendes zur Pathogenität beitragendes Kriterium sein.

Phospholipase-A- und Lysophospholipase-A-Aktivitäten werden vom Typ-II-Protein-Sekretionsapparat sezerniert

Das Typ-II-Sekretionssystem Lsp von L. pneumophila wurde als Virulenzfaktor des Bakteriums charakterisiert [23, 24, 41]. Da, wie bei L. pneumophila und anderen
Bakterien bereits gezeigt, insbesondere hydrolytische Enzyme durch das Typ-IISystem aus der Bakterienzelle geschleust werden, war es von Interesse zu überprüfen, ob auch die Phospholipase-A- und Lysophosphospholipase-A-Aktivitäten Typ-II-sekretiert sind. Durch Vergleich der PLA- und LPLA-Aktivitäten im Kulturüberstand von L.-pneumophila-Wildtyp und einer Mutante mit funktionsunfähigem Sekretionsapparat wurde bewiesen, dass der Export dieser hydrolytischen Enzymaktivitäten vom Typ-II-System abhängt und dass PLA/LPLA-Aktivitäten virulenzvermittelnde Effektoren des Lungenpathogens sein könnten [25, 39, 41].

\section{Die Phospholipase-A-Aktivität zerstört die Phospholipide des Lungensurfactants}

Aufgrund des Vorhandenseins von Phospholipase-Aktivität bei Legionellen liegt z. B. eine Beeinträchtigung der Lungenfunktion über die surfactantzerstörende Wirkung von Phospholipasen nahe. Um dies zu überprüfen, inkubierten wir L.pneumophila-Bakterien oder deren Kulturüberstand in vitro mit Rinderlungensurfactant. Wir stellten fest, dass Fettsäuren freigesetzt, die Hauptsurfactantphospholipide Phosphatidylcholin und Phosphatidylglyzerol reduziert und das zytotoxische Agens Lysophosphatidylcholin gebildet wurden [37]. Lysophosphatidylcholin kann schon in sehr geringen Mengen Lungenepithelzellen lysieren und dadurch eine Durchlässigkeit des alveolären Epithels hervorrufen. Dies kann den Eintritt von Substanzen und Zellen aus dem sich anschließenden Kapillarraum und eine Verstopfung der Alveolen zur Folge haben $[26,27]$. Zusätzlich konnten wir zeigen, dass der Abbau an SurfactantDiazylphospholipiden und die Anreicherung von Lysophosphatidylcholin zur Verschlechterung der physikalischen Eigenschaften des Lungensurfactants führt, die für die Lungenfunktion notwendig sind [37]. Mit diesen Experimenten konnte zum ersten Mal die häufig beobachtete Ausprägung von ARDS und erhöhtem Atemwiderstand bei Patienten mit Legionellenpneumonie erklärt werden [37].

\section{Die maßgebliche Lysophospholipase- A-Aktivität ist eine GDSL-Hydrolase}

Die vorangegangenen Experimente legten die Vermutung nahe, dass es sich bei 


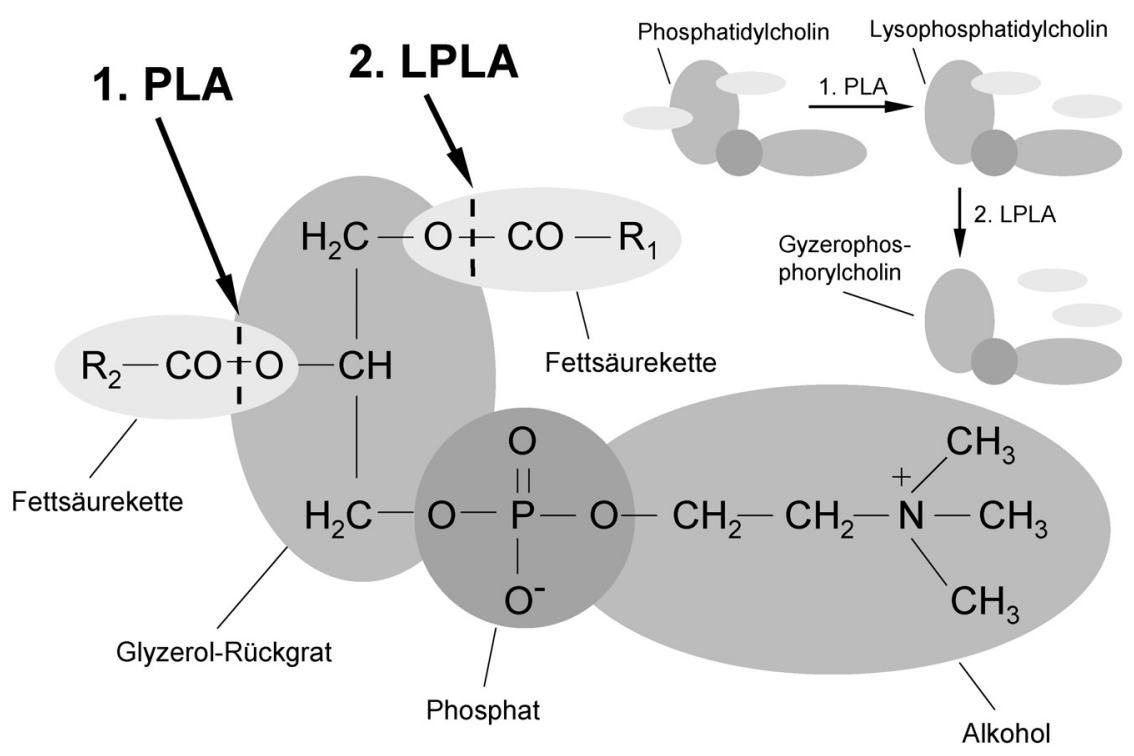

Abb. $4 \Delta$ Aufbau eines Phospholipids (hier Phosphatidylcholin) und zweistufige Hydrolyse durch L.-pneumophila-Enzyme. Phospholipide bestehen aus einem Glyzerol-Rückgrat, an das in sn-1- und sn-2-Stellung jeweils langkettige Fettsäurereste esterartig gebunden sind. An der verbleibenden Hydroxylgruppe des Glyzerol-Rückgrats befindet sich eine Phosphatgruppe, an die ein Alkohol (Cholin im Falle des Phosphatidylcholins) gebunden ist. Phospholipase-A-Aktivität (PLA) von L. pneumophila, die eine Fettsäure aus dem Diazylphospholipid (Phosphatidylcholin) herauslöst und Lysophosphatidylcholin bildet, wird beim Wachstum der Bakterien zeitlich vor der Lysophospholipase-A-Aktivität (LPLA), die eine Fettsäure aus Lysophosphatidylcholin ablöst und damit Glyzerophosphorylcholin erzeugt, sezerniert

den PLA- und LPLA-Aktivitäten von L. pneumophila tatsächlich um Virulenzfaktoren des Lungenpathogens handeln könnte. Aber zu diesem Zeitpunkt waren weder die Protein- noch die Gensequenz der PLA/LPLA-Enzyme bekannt. Wir setzten daher unsere biochemischen Aufreinigungen fort. Im Fall der maßgeblichen LPLA-Aktivität von L. pneumophila konnten wir nach mehreren Aufreinigungsschritten ein $28-29 \mathrm{kDa}$ großes Protein (Bandengröße nach reduzierender SDS-PAGE) N-terminal sequenzieren, das sich in LPLA-Aktivität enthaltenden Chromatographiefraktionen anreicherte [39]. Mithilfe dieser Aminosäuresequenz und der Legionella-Genom-Datenbank (http:// genome3. cpmc.columbia.edu/ legion/ index.html) wurde das LPLA-Gen ( $p l a A=$ Phospholipase $A$ Gen $A$ von L. pneumophila, LPLA ist eine Untergruppe von PLA) aufgefunden, in E. coli kloniert und sequenziert (Genebank Acession No. AF510106). Die abgeleitete PlaA-Proteinsequenz enthält die Erkennungssequenz „GDSL“, die typisch für eine bestimmte Gruppe von lipolytischen Enzymen (Lipasen, Phospholipasen, Acyltransferasen und Hämolysine) ist [39]. Diese Gruppe wird unter der Bezeichnung GDSL-Hydrolasen zusam- mengefasst. Die vor wenigen Jahren neu beschriebene Gruppe der GDSL-Hydrolasen weist 5 aufeinander folgende homologe Proteinregionen (Block I-V) auf [42]. Das Motiv GDSL, das das putative nukleophile Agens Serin (S) enthält, befindet sich in Block I [42]. Die Gruppe der GDSL-Hydrolasen unterscheidet sich in wesentlichen strukturellen Merkmalen von den schon weit reichend erforschten $\alpha / \beta$-Hydrolasen [42]. Mitglieder der GDSL-Hydrolasen findet man bei Gramnegativen oder zellwandlosen Bakterien sowie interessanterweise auch bei höheren Pflanzen ([43], Flieger und Cianciotto unveröffentlichte Beobachtung). Oftmals besitzen bakterielle Pathogene von Pflanzen oder Tieren ein oder wie im Fall von L.pneumophila sogar mehrere Gene, die für ein GDSL-Protein kodieren ([28], Flieger \& Cianciotto unveröffentlichte Beobachtung). Beispiele hierfür sind die Lipase EstA von Pseudomonas aeruginosa, das Lezithin-abhängige Hämolysin von Vibrio cholerae, die Azyltransferase GCAT von Aeromonas hydrophila oder der Typ-III-sekretierte Effektor SseJ von Salmonella typhimurium. Die Enzyme von A. hydrophila und S. typhimurium wurden bereits als Pathogenitätsfaktoren charakterisiert [44, 45].

\section{Die Lysophospholipase A PlaA hydrolysiert Lysophosphatidylcholin und -glyzerol}

Welche katalytische Aktivität besitzt die Lysophospholipase A PlaA von L. pneumophila? Ein plaA-enthaltender E.-coliKlon zeigte eine erhöhte Fettsäurefreisetzung gegenüber Lysophosphatidylcholin als auch gegenüber einem anderen Lysophospholipid, dem Lysophosphatidylglyzerol, jedoch nicht gegenüber Diazylphospholipiden. Kulturüberstände einer L.-pneumophila-plaA-knock-out-Mutante verloren über 90\% ihrer Fähigkeit, Lysophosphatidylcholin zu hydrolysieren. Dies beweist erneut, dass L. pneumophila mit PlaA ein Protein speziell für die Hydrolyse von Lysophospholipiden besitzt. Da sich die Hydrolyse von Lysophosphatidylglyzerol nur um ca. $70-80 \%$ reduzierte, vermuten wir die Anwesenheit von weiteren sekretierten Lysophospholipase-Aktivitäten [28].

\section{Die Lysophospholipase A PlaA spielt eine Rolle bei der Entgiftung von Lysophosphatidylcholin}

Das Protein PlaA von L. pneumophila hydrolysiert Lysophosphatidylcholin. Somit könnte dieses Enzym eine besondere Rolle in der Detoxifikation des hämolytischen Agens spielen, das von bakterieller oder Wirts-Phospholipase A (z. B. aus Lungensurfactant) im Laufe einer Infektion generiert werden könnte. Tatsächlich ist die plaA-Mutante gegenüber Lysophosphatidylcholin empfindlicher als der Wildtyp. Gleichzeitig schützt die Überexpression von plaA das Bakterium vollständig vor der zytolytischen Aktivität des Lysophospholipids [28].

\section{Die Lysophospholipase A PlaA ist für das intrazelluläre Überleben von L. pneumophila entbehrlich}

Da die Virulenz von L. pneumophila in vielen Fällen mit der Fähigkeit korrelliert, Wirtszellen wie Makrophagen oder auch Amöben zu infizieren und sich in diesen $\mathrm{zu}$ vermehren, interessierte uns, ob eine plaA-Mutante des Bakteriums dazu noch in der Lage ist. plaA-Mutanten zeigten eine dem Wildtyp vergleichbare intrazelluläre Überlebens- bzw. Vermehrungsfähigkeit in Amöben sowie in U937-Makrophagen. Dies bedeutet, dass plaA für das Überleben des Pathogens in der Wirtszel- 
le nicht essenziell ist und z. B. durch andere vorhandene Lysophospolipasen A (s. oben) ersetzt werden könnte [28].

\section{Ziele der zukünftigen Forschung}

Da wir bereits wissen, dass L. pneumophila mehrere Phospholipasen A/Lysophospholipasen A produziert, sollen in $\mathrm{Zu}$ kunft die entsprechenden Gene aufgefunden und die Proteine im Hinblick auf ihren Beitrag zur Pathogenität von Legionellen erforscht werden. Zu diesem Zweck sind biochemische Aufreinigungen von L.-pneumophila-Zellkulturüberstand geplant. Proteine, die mit einer Phospholipase-Aktivität assoziieren, sollen $\mathrm{N}$-terminal sequenziert und die entsprechenden Gene mithilfe der Genomdatenbank für L. pneumophila identifiziert werden. Anschließend sollen diese Gene in L. pneumophila deletiert und die resultierenden Mutanten auf ihre intrazelluläre Vermehrung in Wirtszellen sowie auf ihren zytopathischen Effekt für eukaryonte Zellen im Vergleich zum Wildtyp analysiert werden. Weiterhin sind Untersuchungen zur Inhibition dieser hydrolytischen Enzyme mit dem Ziel der Entwicklung neuer therapeutischer Ansätze zur Behandlung einer Legionellose und anderer durch bakterielle Phospholipasen potenzierte Pneumonien geplant. Von Interesse ist auch, Zellkultursysteme zu entwickeln, die dem tatsächlichen in der Lunge vorhandenen Milieu nahe sind, d. h., es sollen Systeme etabliert werden, die den Verlauf der Lungeninfektion durch L. pneumophila in vitro exakter als die bisherigen Zellkultursysteme widerspiegeln. In einem weiteren Projekt versuchen wir, die Rolle von Phospholipasen der Wirtszelle an der Entwicklung einer Legionellenpneumonie zu bestimmen.

Danksagung Wir bedanken uns bei Gudrun Holland und Dr. Muhsin Özel (Robert KochInstitut, Berlin) für die Ausführung der Elektronenmikroskopie sowie allen anderen Mitarbeitern und Mitarbeiterinnen des Robert Koch-Instituts für eine gute Zusammenarbeit. Außerdem gilt unser Dank PD Dr. Birgid Neumeister, Marion Faigle, PD Dr. Stefan Stevanovic, Dr. Elvira Fehrenbach (Universität Tübingen) und Nicholas P. Cianciotto (Northwestern University Chicago) für die enge Kooperation bei der Charakterisierung derLegionellaPhospholipase-A-Enzyme sowie für die Bereitstellung von Bakterien- und Wirtszellisolaten. Weiterhin danken wir Heinz Reinhardt (Boehringer Ingelheim KG, Biberach) für die Bereitstellung von Rinderlungensurfactant so- wie Josef Mußotter, Eberhard Weller, Prof. Dr. Dr. Peter Bartmann (Universität Bonn), Dr. Ulf Kehrer und Prof. Dr. Hermann A. Mayer (Universität Tübingen) für ihre Unterstützung bei den Experimenten mit Lungensurfactant bzw. der Lungensurfactantanalytik.

\section{Literatur}

1. McDade JE, Shepard CC, Fraser DW et al. (1977) Legionnaires' disease: isolation of a bacterium and demonstration of its role in other respiratory disease. $\mathrm{N}$ Engl J Med 297:1197-1203

2. Fraser DW, Tsai TR, Orenstein W et al. (1977) Legionnaires' disease: description of an epidemic of pneumonia.N Engl J Med 297:1189-1197

3. McDade JE, Brenner DJ, Bozeman M (1979) Legionnaires' disease bacterium isolated in 1947. Ann Int Med 90:659-661

4. Cianciotto NP (2001) Pathogenicity of Legionella pneumophila. Int J Med Microbiol 291:331-343

5. Rogers J, Keevil CW (1992) Immunogold and fluorescein immunolabelling of Legionella pneumophila within an aquatic biofilm visualized by using episcopic differential interference contrast microscopy. Appl Environ Microbiol 58:2326-2330

6. Fields BS (1996) The molecular ecology of legionellae. Trends Microbiol 4:286-290

7. Brieland JK, Fantone JC, Remick DG et al. (1997) The role of Legionella pneumophila-infected Hartmannella vermiformis as an infectious particle in a murine model of Legionnaire's disease. Infect Immun 65:5330-5333

8. Pedro-Botet ML, Sabria-Leal M,Sopena N et al. (1998) Role of immunosuppression in the evolution of Legionnaires' disease. Clin Infect Dis 26:14-19

9. Edelstein PH (1993) Legionnaires' disease. Clin Infect Dis 16:741-749

10. Winn WC Jr, Davis GS, Gump DW et al. (1982) Legionnaires' pneumonia after intratracheal inoculation of guinea pigs and rats. Lab Invest 47:568-578

11. Waanders H, Dik H, Meinders AE (1991) Legionella pneumonia complicated by adult respiratory distress syndrome. Neth J Med 38:159-166

12. Fang GD, Fine M, Orloff J et. al (1990) New and emerging etiologies for community-acquired pneumonia with implications for therapy. A prospective multicenter study of 359 cases. Medicine Baltimore 69:307-316

13. Robert Koch-Institut (2003) Aktuelle Statistik meldepflichtiger Infektionskrankheiten. Epidemiolog Bull18:144

14. Horwitz MA (1983) Formation of a novel phagosome by the Legionnaires' disease bacterium (Legionella pneumophila) in human monocytes.J Exp Med 158:1319-1331

15. Horwitz MA (1983) The Legionnaires' disease bacterium (Legionella pneumophila) inhibits phagosome-lysosome fusion in human monocytes.J Exp Med 158:2108-2126

16. Sturgill-Koszycki S, Swanson MS (2000) Legionella pneumophila replication vacuoles mature into acidic, endocytic organelles.J Exp Med 192:1261-1272

17. Swanson MS, Hammer BK (2000) Legionella pneumophila pathogesesis: a fateful journey from amoebae to macrophages. Annu Rev Microbiol 54:567-613

18. Wright JR, Clements JA (1987) Metabolism and turnover of lung surfactant. Am Rev Respir Dis 136:426-444

19. Pison U,SeegerW, Buchhorn R et al.(1989) Surfactant abnormalities in patients with respiratory failure after multiple trauma. Am Rev Respir Dis 140:1033-1039

20. Gunther A, Siebert C, Schmidt R et al. (1996) Surfactant alterations in severe pneumonia, acute respiratory distress syndrome, and cardiogenic lung edema. Am J Respir Crit Care Med 153:176-184

21. Marra AS, Blander J, Horwitz MA, Shuman HA (1992) Identification of a Legionella pneumophila locus required for intracellular multiplication in human macrophages. Proc Natl Acad Sci USA 89:9607-9611

22. Berger KH, Isberg RR (1993) Two distinct defects in intracellular growth complemented by a single genetic locus in Legionella pneumophila. Mol Microbiol 7:17-19

23. Hales LM, Shuman HA (1999) Legionella pneumophila contains a type II general secretion pathway required for growth in amoebae as well as for secretion of the Msp protease.Infect Immun 67:3662-3666
24. Liles MR, Edelstein PH, Cianciotto NP (1999) The prepilin peptidase is required for protein secretion by and the virulence of the intracellular pathogen Legionella pneumophila. Mol Microbiol 31:959-970

25. Rossier 0, Cianciotto NP (2001) Type II protein secretion is a subset of the PilD-dependent processes that facilitate intracellular infection by Legionella pneumophila. Infect Immun 69:2092-2098

26. Weltzien HU (1979) Cytolytic and membrane-perturbing properties of lysophosphatidylcholine. Biochim Biophys Acta 559:259-287

27. Niewoehner DE, Rice K, Sinha AA, Wangensteen D (1987) Injurious effects of lysophosphatidylcholine on barrier properties of alveolar epithelium.J Appl Physiol 63:1979-1986

28. Smith GA, Marquis H, Jones S et al. (1995) The two distinct phospholipases C of Listeria monocytogenes have overlapping roles in escape from a vacuole and cell-tocell spread. Infect Immun 63:4231-4237

29. Plotkowski MC, Meirelles MN (1997) Concomitant endosome-phagosome fusion and lysis of endosomal membranes account for Pseudomonas aeruginosa survival in human endothelial cells.J Submicrosc Cytol Patho 29:229-237

30. Dennis EA (1997) The growing phospholipase A2 superfamily of signal transduction enzymes. Trends Biochem Sci 22:1-2

31. Prokazova NV, Zvezdina ND, Korotaeva AA (1998) Effect of lysophosphatidylcholine on transmembrane signal transduction. Biochemistry Mosc 63:31-37

32. Kume N, Cybulsky MI, Gimbrone MA Jr (1992) Lysophosphatidylcholine, a component of atherogenic lipoproteins, induces mononuclear leukocyte adhesion molecules in cultured human and rabbit arterial endothelial cells.J Clin Invest 90:1138-1144

33. Masamune A, Sakai Y, Satoh A et al. (2001) Lysophosphatidylcholine induces apoptosis in AR42 $\mathrm{J}$ cells. Pancreas 22:75-83

34. Lema G, Dryja D, Vargas I, Enhorning G (2000) Pseudomonas aeruginosa from patients with cystic fibrosis affects function of pulmonary surfactant. Pediatr Res 47:121-126

35. Holm BA, Keicher L, Liu MY et al. (1991) Inhibition of pulmonary surfactant function by phospholipases.J Appl Physiol 71:317-321

36. Flieger A, Gong S, Faigle $M$ et al. Novel phospholipase $A$ (PLA) activity secreted by Legionella species.J Bacteriol 182:1321-1327

37. Flieger A, Gong S, Faigle M et al. (2000) Phospholipase A secreted by Legionella pneumophila destroys alveolar surfactant phospholipids. FEMS Microbiol Lett 188:129-133

38. Flieger A, Neumeister B, Cianciotto NP (2002) Characterization of the gene encoding the major secreted lysophospholipase A of Legionella pneumophila and its role in detoxification of lysophosphatidylcholine. Infect Immun 70:6094-6106

39. Flieger A, Gong S, Faigle M et al. (2001) Novel lysophos pholipase A secreted byLegionella pneumophila.J Bac teriol 183:2121-2124

40. Flieger A, Gong S, Faigle M et al. (2001) In vitro secretion kinetics of proteins from Legionella pneumophila in comparison to proteins from non-pneumophila species. Microbiology 147:3127-3134

41. Aragon V, Kurtz S, Flieger A et al. (2000) Secretion activities of wild type and pilD deficient Legionella pneumophila. Infect Immun 68:1855-1863

42. Upton C, Buckley JT (1995) A new family of lipolytic enzymes? Trends Biochem Sci 20:178-179

43. Brick DJ, Brumlik MJ, Buckley JT et al. (1995) A new family of lipolytic plant enzymes with members in rice, arabidopsis and maize. FEBS Lett 377:475-480

44. Lee KK, Ellis AE (1990) Glycerophospholipid:cholesterol acyltransferase complexed with lipopolysaccharide (LPS) is a major lethal exotoxin and cytolysin of Aeromonas salmonicida: LPS stabilizes and enhances toxicity of the enzyme.J Bacteriol 172:5382-5393

45. Ruiz-Albert J, Yu XJ, Beuzon CR et al. (2002) Complementary activities of SseJ and SifA regulate dynamics of the Salmonella typhimurium vacuolar membrane. Mol Microbiol 44:645-661 